\title{
Exercise Regulates Peripheral Blood Serotonin to Improve the Addiction and Impulse of Methamphetamine Withdrawal Patients
}

\section{Wanke Tang}

Hunan Normal University

Lan Zheng ( $\nabla$ hnsd2017wjs@163.com )

Hunan Normal University

\section{Research Article}

Keywords: exercise, 5-HT, craving, impulse

Posted Date: September 16th, 2021

DOI: https://doi.org/10.21203/rs.3.rs-800259/v1

License: (c) (1) This work is licensed under a Creative Commons Attribution 4.0 International License.

Read Full License 


\section{Exercise regulates peripheral blood serotonin to improve the addiction and}

impulse of methamphetamine withdrawal patients

Wanke Tang $^{1 \#} ;$ Lan Zheng ${ }^{1 *}$

1: Hunan Normal University

Province: Hunan Judicial Police Vocational College

First Author:Name: Wanke Tang E-mail: 912079965@qq.com

Correspondence Author: Lan Zheng E-mail:lanzheng@hunnu.edu.cn

Abstract: Background: This study aimed to explore the effect of aerobic combined with resistance exercise on the level of 5-HT in the peripheral blood of methamphetamine $(\mathrm{METH})$ withdrawal patients, improving cue-induced craving and impulse.

Methods : Fifty methamphetamine withdrawal patients were included. The patients underwent eight weeks of aerobic combined with resistance exercise. The sessions lasted an hour and were conducted five times a week. Impulsivity (BIS-11), craving (VAS craving), and control (VAS control) were assessed before and after the intervention. ELISA was used to detect peripheral blood 5-HT levels.

Results : The results showed that peripheral blood 5-HT and VAS control significantly increased after the exercise. In contrast, VAS craving and BIS-11 significantly decreased after the exercise.

Conclusions : We present that eight weeks of aerobic combined with resistance exercise can alter the peripheral blood 5-HT levels of METH withdrawal patients and improve the cue-induced craving and impulse levels.

Keyword: exercise; 5-HT; craving; impulse

Advantages and limitations:

In this work, we explored the relationship between peripheral 5-HT and addiction and impulsivity in methamphetamine withdrawal patients. Interestingly, they have a high correlation between them. And the linear regression is significant. At the same time, we found that exercise as an external factor can play a certain role in improvement. As far as we know, this study is a prospective human experiment, and most of the previous studies were animal studies. This article alone has certain limitations. Peripheral 5-HT cannot pass through the blood-brain barrier. After all, it cannot 
represent the condition of the central nervous system. At the same time, the number of subjects is small and the duration of exercise intervention is relatively short, which affects the effect of the data to a certain extent.

\section{Introduction}

Dopaminergic and glutamatergic neural circuits have been reported to play a significant role in the neuroplasticity of addiction in recent years. Besides, the serotonergic mechanism promotes addiction. Methamphetamine (METH) and other amphetamine derivatives can release 5-HT, which causes degenerative changes in 5-HT axons throughout the cerebral cortex, striatum, and hippocampus [1-3]. Serotonin (5-HT) plays a mediating role in neurotoxicity and addiction induced by METH [4]. METH can damage the 5-HT system regulating impulsive behaviour, causing neurotoxicity, changing impulsive behaviour, and thus affecting the development of addiction [5].

Serotonin $(5-\mathrm{HT})$ is a monoamine neurotransmitter. Most peripheral 5-HT is found in platelets, while a small amount is found in cell-free plasma [6]. Moreover, 5-HT involves various cognitive and behavioural responses. For instance, 5-HT plays a crucial role in impulsive responses [7]. Impulsive features in mammals involve the monoaminergic system of the midbrain, including 5-HT neurons, which form the basis of the complex interaction between the prefrontal lobe and the orbital cortex and the basal ganglia [8-9]. Impulse is essential for the development, maintenance, and recurrence of drug addiction [10]. Impulse mainly includes lack of thought before action, sensory seeking, motor and cognitive impulse behaviour, and poor ability to 
delay gratification [11-12].

A good impulse is associated with the best decisions. However, a high level of impulse can lead to undesirable consequences, such as drug addiction [13]. Studies have shown that SUD individuals who use stimulants, opiates, and alcohol have a higher level of impulsivity than the non-psychotic controls [14]. Similarly, some scholars believe that impulsivity is a potential marker of SUD [15]. Therefore, further research is needed to assess whether impulsivity can be an effective treatment for SUD.

Drug abuse can cause certain cognitive impairments in the brain. However, exercise can improve memory, inhibition, control, and attention in adults with mild cognitive impairment [16]. Besides, exercise can treat drug addiction and relapse [17]. Eight weeks of aerobic combined with resistance exercise can reduce anxiety and depression of METH withdrawal patients, reducing the use of METH after discharge from the hospital. Several [18-20]studies have shown that aerobic exercise can reduce the related cravings of METH-dependent individuals, enhancing inhibitory control [21]. Although many studies have proven that exercise can improve impulse or addiction, its peripheral biological mechanism is unknown. This prospective study aimed to explore the role of peripheral 5-HT in impulsiveness and cue-induced cravings of METH withdrawal patients. Therefore, this study can provide a theoretical reference for the diagnosis and treatment of drug addiction and relapse.

\section{Research objects and methods}

\section{Research subjects}

A total of 960 males from compulsory drug rehabilitation centres in Bainihu Compulsory Isolation and Detoxification Center in Hunan Province were used as the screening subjects. The selection criteria were: 1) All participants underwent structured diagnostic interviews based on the "Diagnosis and Statistics Manual of Mental Disorders-5th Edition". The participants had to meet the MA dependence standards. In addition, they had: 2) No mental illness; 3) No bone and muscle disease, cardiovascular, cerebrovascular, and immune disease; 4) No history of major trauma 
and surgery; 5) No exercise habits (three Moderate-intensity physical activity for less than 30 minutes a day and less than three days a week for three months) [22]. Lastly, the participants filled the PAR-Q+ (physical activity preparation questionnaire) to ensure they were suitable for the exercise.See Figure 1 for the screening process.

This research was conducted following the Helsinki declaration, and it (number: 2020-233) was approved by the Review Committee of the Ethics Institution of Hunan Normal University. Fifty subjects volunteered to participate in the trial and signed an informed consent form. The subjects were randomly divided into control and exercise groups ( 25 each) after the baseline test. Two subjects withdrew from the control group (withdrawal rate, $8 \%$ ), and three from the exercise group (withdrawal rate, $12 \%$ ). The basic conditions of the subjects are shown in Table $\mathbf{1 .}$

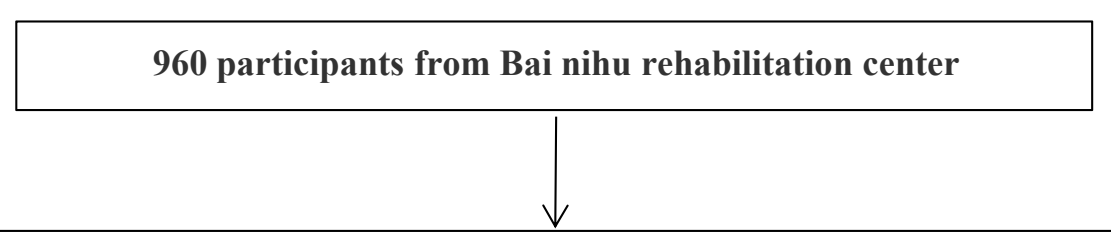

Filling the basic-case questionnaire, determining METH drug type usage. A total of 560 people were screened $(58.3 \%)(560 / 960)$.

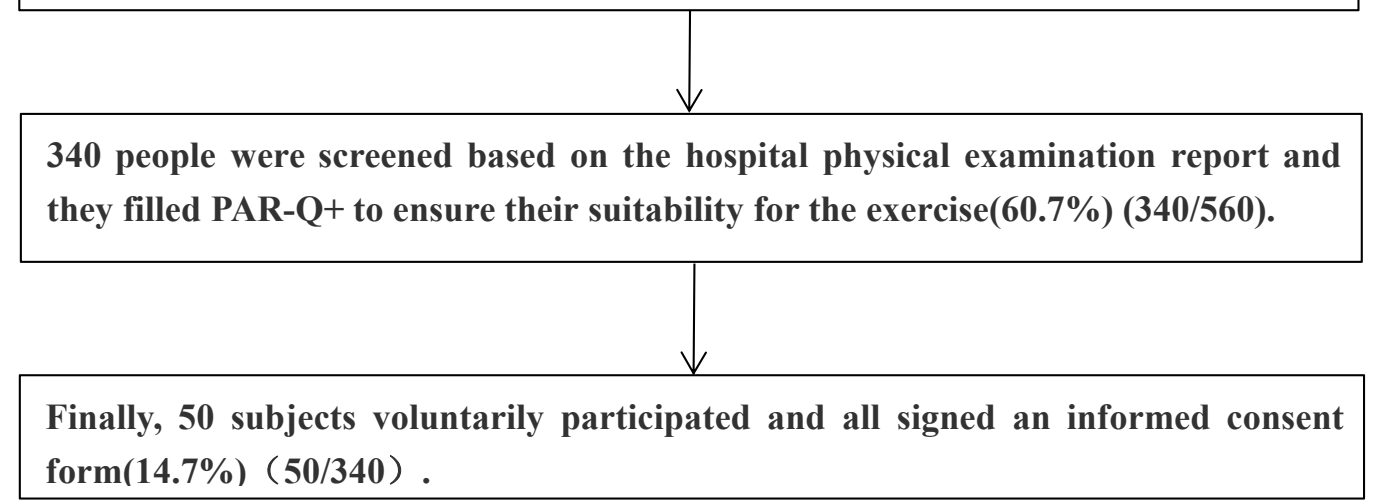

FIGURE 1: A flowchart of the participants screening process 
Table 1 The Basic Situation of Research Objects

\begin{tabular}{lcc}
\hline Variable & Control group $(n=22)$ & Exercise group $(n=22)$ \\
\hline Age(years $)$ & $29.83 \pm 5.96$ & $32.86 \pm 5.56$ \\
Height $(\mathrm{cm})$ & $165.60 \pm 31.12$ & $171.34 \pm 5.79$ \\
Culture & & \\
Primary School or less & $7(31.8)$ & $7(31.8)$ \\
Junior high school & $6(27.3)$ & $8(36.3)$ \\
Senior middle school & $9(40.9)$ & $5(22.7)$ \\
College or above & $1(4.5)$ & $2(9.1)$ \\
Occupation & & $5(22.7)$ \\
Unemployed & $8(36.3)$ & $5(22.7)$ \\
Self-employed & $3(13.6)$ & $4(18.2)$ \\
Service & $1(4.5)$ & $1(4.5)$ \\
Manual Worker & $3(13.6)$ & $1(4.5)$ \\
General Staff & $3(13.6)$ & $2(9.1)$ \\
Other & $4(18.2)$ & $5(22.7)$ \\
Marital Status & $7(31.8)$ & \\
Married & & \\
\hline & & \\
\hline
\end{tabular}




\begin{tabular}{lcc}
\hline Single & $6(27.3)$ & $10(45.5)$ \\
Widowed & $1(4.5)$ & $5(22.7)$ \\
Divorced & $8(36.4)$ & $2(9.1)$ \\
Regions & & $10(45.5)$ \\
Urban & $10(45.5)$ & $12(55.5)$ \\
Rural & & \\
Drug data & $0.56 \pm 0.40$ & $0.51 \pm 0.42$ \\
Heroin use at one time $(\mathrm{g})$ & $13.98 \pm 9.64$ \\
Times per month & $12.36 \pm 9.42$ & $96.41 \pm 34.20$ \\
Duration (months) & $87.45 \pm 65.33$ & \\
\hline
\end{tabular}

\section{Exercise intervention}

The American College of Sports Medicine (ACSM) recommends an exercise intervention combining aerobic exercise and resistance training ("Abstracts from the 37th Annual Meeting of the Society of General Internal Medicine, 2014, San Diego, CA, USA," 2014). Participants in the control group received routine nursing work in the drug rehabilitation centre and did not participate in any sports. The subjects in the exercise group received structured and progressive exercises following the FITT-VP (Frequency, Intensity, Time, Type) mode of the American Society of Sports Medicine. The maximum heart rate (206.9-0.67×age) and weight (1RM) of each person were determined before each exercise cycle. The exercise intervention was conducted five times a week for eight weeks (60 minutes for each exercise). Besides, a 5-minute warm-up exercise, 30-minute treadmill aerobic training, 20-minute strength resistance training (arms, chest, back, buttocks, legs, and core muscles), and a 5-minute stretching and relaxation training were conducted. Resistance training involves core muscles and lower limb muscles. Strength resistance training improves muscle strength and explosive power. Each set was conducted 8-12 times at a 60-second interval.See Figure 2 for the flow chart of exercise intervention.

Type of exercise: Aerobic combined resistance exercise with increasing load. Duration of exercise: $90 \mathrm{~min} /$ day; 5 day/week; 8 weeks. Exercise program: $5 \mathrm{~min}$ warm-up exercise, 30minute treadmill aerobic training, 20min strength resistance training, $5 \mathrm{~min}$ stretching and relaxation training. 


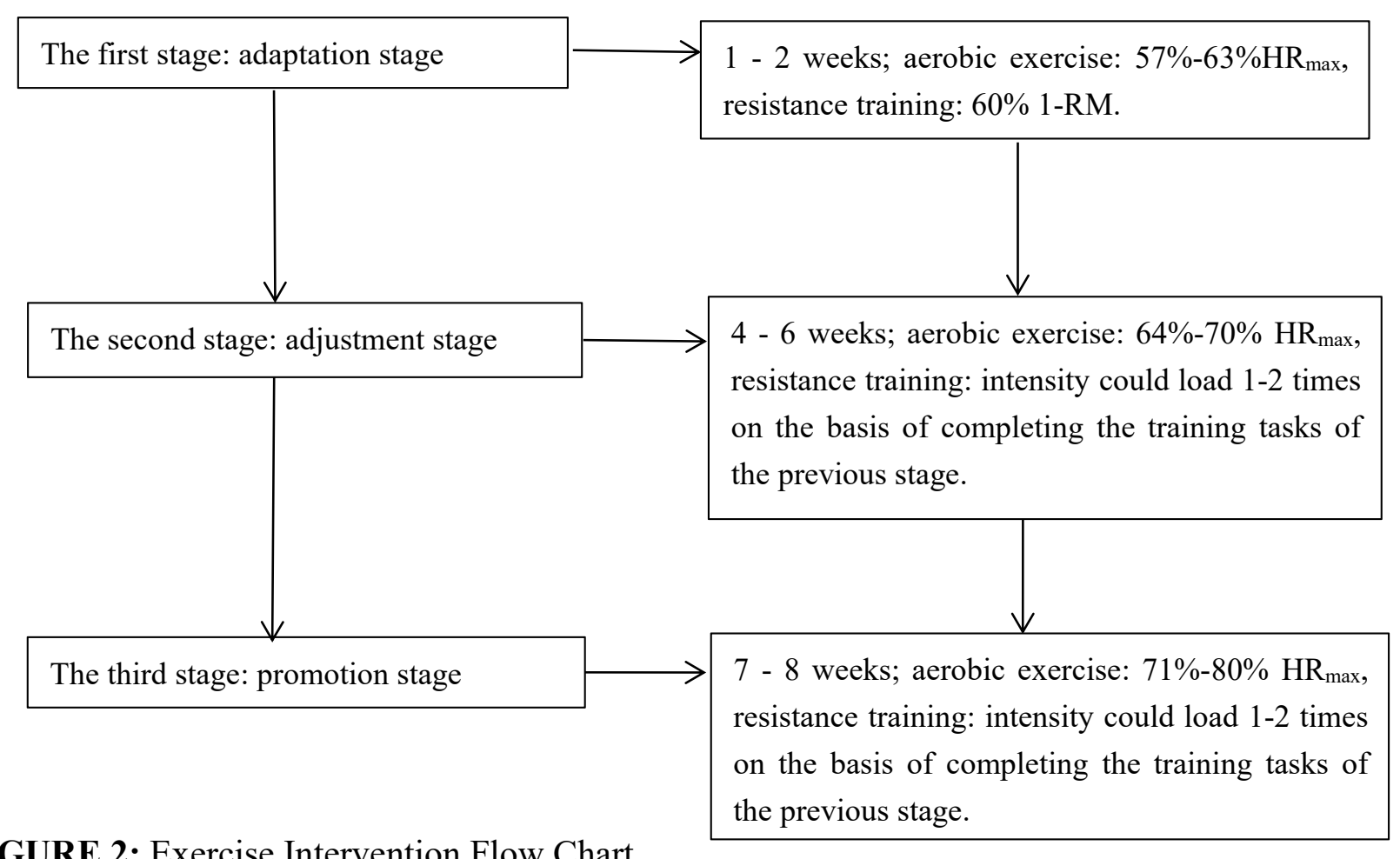

FIGURE 2: Exercise Intervention Flow Chart

\section{PAR-Q(Physical Activity Readiness Questionnaire)}

PAR-Q provides a safe initial screening of candidates for exercise testing and exercise prescriptions, ensuring that the subjects are suitable for sports activities.

\section{BIS-11 (Barratt Impulsiveness Scale)}

Barratt-11 has 26 items with three dimensions: attention impulsivity, motor impulsivity, and unplanned impulsivity. A 4-level scoring method (1-4 points) was used for scoring. The higher the score, the stronger the impulsivity. The Cronbachs' alpha coefficient of the Chinese version of BIS-11 was 0.759 .

\section{Visual Analog Scale (VAS)}

VAS is often used to measure subjective feelings. Herein, VAS craving and VAS control were used to evaluate the cue-induced craving and self-control ability. The scale was scored from 0-100 points, where 0 indicates no drug desire and no sense of self-control and 100 represents a high level of drug desire and a high sense of self-control. Participants indicate how they feel by making a mark on the horizontal line. The images, objects and videos about METH were observed for five minutes prior to answering the following questions. "What is your craving for drugs at this moment?" and "What is your sense of self-control over drug use at this moment?"

\section{Enzyme-linked immunosorbent assay (ELISA)}

Blood samples were collected from 7:00 to 8:00 a.m. daily before and after the exercise intervention. The subjects were starved from 8 p.m. the day before the blood collection. Venous blood $(2 \mathrm{ml})$ was collected in an ADTA anticoagulant test tube, mixed with an anticoagulant, and centrifuged at 4000rpm for four minutes. The supernatant was stored in an ultra-low temperature refrigerator at $-80{ }^{\circ} \mathrm{C}$ for further analysis. A Shanghai Enzyme Association assessed the blood indicators. 


\section{Statistical analysis}

The demographic data are expressed as mean \pm standard deviation $(\mathrm{M} \pm \mathrm{SD})$, frequency, and percentage. The normality was assessed by testing the normal plot of the residuals in the analysis of the variance model. A mixed analysis of variance was used for comparison between multiple groups. Bonferroni method was used for pairwise comparison between groups $(P<0.05)$. SPSS20.0 software was used for statistical analysis. GraphPad Prism 5 (GraphPad Software, San Diego, CA, USA) was used for graphic presentations.

\section{Results}

\section{The effect of exercise on peripheral blood 5-HT}

The mixed analysis of variance method was used to analyze the effects of exercise and time on peripheral blood 5-HT. The results showed that the interaction effects of time and time* group altered peripheral 5-HT $(p<0.001)$. The peripheral 5-HT was not significantly different between the control and the exercise groups before exercise $(p>0.05)$. However, the 5-HT level was significantly lower in the control group than in the exercise group after exercise $(p<0.01)$ (TABLE 2).

TABLE 2: The effect of exercise on 5-HT

\begin{tabular}{|l|l|l|l|l|l|}
\hline Group & $\begin{array}{l}\text { Pre-exercise } \\
\mathbf{( p g} / \mathbf{m l})\end{array}$ & $\begin{array}{l}\text { Post-exercise } \\
\mathbf{( p g / m l )}\end{array}$ & Intergroup & Time & Interaction \\
\hline Control group & $226.15 \pm 29.65$ & $228.96 \pm 27.66$ & $p=0.196$ & $p<0.001$ & $p<0.001$ \\
\hline Exercise group & $220.35 \pm 28.22$ & $\begin{array}{l}255.35 \pm 24.54 \\
\triangle \triangle * *\end{array}$ & $\mathrm{~F}=1.728$ & $\mathrm{~F}=37.21$ & $\mathrm{~F}=27.05$ \\
& & & & \\
\hline
\end{tabular}

$\triangle \triangle p<0.01$ compared with the same time point of Control group; $* * p<0.01$ compared with the same group at pre-exercise.

\section{Exercise improves VAS craving}

The mixed analysis of variance method was also used to analyze the influence of group and time on VAS craving. The results showed that the interaction effect of time and time* group affected VAS craving $(p<0.05)$. The VAS craving was not significantly different between the control and the exercise group before exercise $(p>0.05)$. However, the VAS craving was significantly reduced in the exercise group than in the control group after exercise $(p<0.01)$ (TABLE 3).

TABLE 3: The effect of exercise on VAS craving

\begin{tabular}{|l|l|l|l|l|l|}
\hline Group & Pre-exercise & Post-exercise & Intergroup & Time & Interaction \\
\hline Control group & $41.73 \pm 27.47$ & $41.68 \pm 23.24$ & $p=0.359$ & $p<0.05$ & $p<0.05$ \\
Exercise group & $40.64 \pm 27.65$ & $29.91 \pm 18.54$ & $\mathrm{~F}=0.861$ & $\mathrm{~F}=4.41$ & $\mathrm{~F}=4.33$ \\
& & $* *$ & & & \\
& & & &
\end{tabular}

$* * p<0.01$ compared with the same group at pre-exercise.

\section{Exercise improves VAS control}

The mixed analysis of variance method was used to analyze the effect of group and time on VAS control. The results showed that: the interaction effect of time and time* group affected VAS control $(p<0.05)$. The VAS control was not significantly different between the control group and the exercise group before exercise $(p>0.05)$. 
In contrast, VAS control was significantly enhanced in the exercise group than in the control group after exercise $(p<0.01)$ (TABLE 4).

TABLE 4: The effect of exercise on VAS control

\begin{tabular}{|l|l|l|l|l|l|}
\hline Group & Pre-exercise & Post-exercise & Intergroup & Time & Interaction \\
\hline Control group & $22.86 \pm 18.06$ & $24.45 \pm 16.61$ & $p=0.127$ & $p=0.089$ & $p<0.05$ \\
Exercise group & $27.00 \pm 17.61$ & $35.91 \pm 19.19$ & $\mathrm{~F}=2.42$ & $\mathrm{~F}=3.04$ & $\mathrm{~F}=6.52$ \\
& & $\triangle_{* *}$ & & \\
& & & &
\end{tabular}

$\triangle p<0.05$ compared with the same time point of Control group; $* * p<0.01$ compared with the same group at pre-exercise.

\section{Exercise improves impulse}

The mixed analysis of variance method was used to analyze the influence of group and time on impulse. The results found that the interaction effects of group, time, and time* group improved impulse $(p<0.05)$. The impulse was not significantly different between the control group and the exercise group before exercise $(p>0.05)$. However, the impulse was significantly reduced in the exercise group than in the control group after exercise $(p<0.01)$ (TABLE 5).

TABLE 5: The effect of exercise on implus

\begin{tabular}{|l|l|l|l|l|l|}
\hline Group & Pre-exercise & Post-exercise & Intergroup & Time & Interaction \\
\hline Control group & $70.41 \pm 6.09$ & $70.55 \pm 5.03$ & $p<0.001$ & $p<0.01$ & $p<0.01$ \\
Exercise group & $69.55 \pm 6.71$ & $60.27 \pm 7.47$ & $\mathrm{~F}=15.86$ & $\mathrm{~F}=11.78$ & $\mathrm{~F}=11.11$ \\
& & & & & \\
& & & & &
\end{tabular}

$\triangle \triangle p<0.01$ compared with the same time point of Control group; ${ }^{*} p<0.01$ compared with the same group at pre-exercise.

\section{Correlation analysis among 5-HT, VAS craving, VAS control, and impulse}

Pearson correlation analysis was used to analyze the correlation among 5-HT, VAS craving, VAS control, and impulse. The results found that 5-HT was negatively correlated with VAS craving $(\mathrm{r}=-0.549, p<0.001)$ and impulse $(\mathrm{r}=-0.393, p<0.01)$ and positively correlated with VAS control $(\mathrm{r}=0.483, p<0.01)$. VAS craving was positively correlated with impulse $(\mathrm{r}=-0.660, p<0.001)$ and negatively correlated with VAS control $(\mathrm{r}=0.567, p<0.001)$. VAS control was negatively correlated with impulse $(\mathrm{r}=-0.576, p<0.001)($ TABLE 6$)$.

TABLE 6: Correlation analysis between 5-HT and VAS craving, VAS control, impulse

\begin{tabular}{|l|l|l|l|l|}
\hline Dimension & 5-HT & VAS craving & VAS control & Implus \\
\hline 5-HT & $r=1$ & $r=-0.549^{* *}$ & $r=0.483^{* *}$ & $r=-0.393^{* *}$ \\
VAS craving & & $r=1$ & $r=0.567^{* *}$ & $r=-0.660^{* *}$ \\
VAS control & & & $r=1$ & $r=-0.576^{* *}$ \\
Implus & & & & $r=1$ \\
\hline
\end{tabular}

\section{Discussion}

Herein, exercise regulated peripheral 5-HT levels and improved addiction and 
impulsivity. This is the first study using human subjects to assess the effect of peripheral 5-HT on addiction and impulsivity of METH withdrawal patients. The study provides insights into peripheral biomarkers of addiction and impulsivity to increase the accuracy of diagnosis and treatment.

\section{The relationship among 5-HT, addiction, and impulsivity in METH withdrawal patients}

Clinical experiments have shown that addiction is correlated with a lack of impulse control. High levels of impulse are considered a risk factor for substance use disorders and may lead to all aspects of the addiction cycle [23]. Besides, 5-HT significantly promotes the vulnerability of addiction. 5-HT also promotes impulse [24]. METH can penetrate the blood-brain barrier and enter the central nervous system of the brain. This damages the 5-HT system, producing neurotoxicity [5], and affecting the development of addiction. Herein, a baseline assessment of the impulsivity level of the existing personnel was conducted. The mean value of BIS-11 in the control group (70.41 \pm 6.09$)$ and exercise group $(69.55 \pm 6.71)$ was in the range of high impulsivity [25] ( $\geq 72$ points). Besides, the average craving degree was above 40 points, indicating moderate craving [26] (40-60 points). Although the number of subjects was small, which can affect the completeness of data interpretation, the consumption of METH was associated with significant subjective impulsive responses and cue-induced cravings.

Previous researches focused on the role of 5-HT in the central nervous system (CNS) since 5-HT is mostly found in the central nervous system. Serotonin drugs have been used to treat various mental disorders, such as depression and obsessive-compulsive disorder [28]. However, 5-HT also promotes peripheral blood circulation. Most 5-HT in peripheral blood is found in platelets, and a small amount is found in cell-free plasma [31]. A human pathological gambling study showed that serotonin function could change due to decreased peripheral 5-HT levels [29]. Besides, peripheral 5-HT reuptake transporter is decreased in pathological gamblers [30]. These studies are based on the uncertain relationship between plasma 5-HT and brain 5-HT [32-33]. However, it is unknown whether the level of 5-HT in peripheral blood is associated with the desire and impulse of METH withdrawal. Herein, 5-HT was negatively correlated with VAS craving and impulse and positively correlated with VAS control, further validating our hypothesis. The low correlation with impulse could be because VAS is a single-dimensional measurement, with less error in the evaluation process, and impulse has to be evaluated in multiple dimensions.

\section{The effect of exercise on 5-HT, VAS craving, VAS control, impulse}

Exercise can improve METH-dependent emotional state, cognitive control, and cravings [34]. Herein, eight weeks of aerobic combined with resistance exercise significantly reduced the anxiety and depression symptoms of METH withdrawal [35]. Also, the exercise improved the heart rate variability of METH addicts. [36]. Taken together, these results indicate that exercise can improve the defects of drug addicts. Moreover, exercise increased the peripheral Blood 5-HT levels, similar to animal studies [37]. 


\section{Conclusion}

Eight weeks of aerobic combined with resistance exercise can regulate the peripheral 5-HT levels of METH withdrawal patients and positively affect cue-induced craving and impulse.

\section{DATA AVAILABILITY STATEMENT}

All datasets generated for this study are included in the article.

\section{COMPETING INTEREST}

No authors note any direct competing interest.

\section{AUTHOR'S CONTRIBUTIUON}

Lan Zheng, Wanke Tang conceived and designed the experiments. screened experimental subjects, Wanke Tang signed the informed consent process and conducted the exercise intervention.

\section{ETHICS STATEMENT}

The Ethics Committee of Hunan Normal University approved this study. All participants signed an informed consent form before conducting the experiment, which was to participate in the training voluntarily.

\section{CONSENT FOR PUBLICATION}

All authors agree to publish the manuscript in this journal

\section{FUNDING}

This research is supported by the National Key Research and Development Program (2016YFC0800908).

\section{ACKNOWLEDGMENTS}

We declare that this work was conducted without any commercial or financial relationships that could be construed as a potential conflict of interest. Thanks to the Bai Nihu Compulsory Isolation and Drug Rehabilitation Center of Hunan Province for the help of the child labor in this experiment.

\section{Reference}

[1]O'Hearn E, Battaglia G, De Souza EB, Kuhar MJ, Molliver ME. Methylenedioxyamphetamine (MDA) and methylenedioxymethamphetamine (MDMA) cause selective ablation of serotonergic axon terminals in forebrain: immunocytochemical evidence for neurotoxicity. J Neurosci. 1988;8(8):2788-2803. 
doi:10.1523/JNEUROSCI.08-08-02788.1988

[2]Molliver ME, Berger UV, Mamounas LA, Molliver DC, O'Hearn E, Wilson MA. Neurotoxicity of MDMA and related compounds: anatomic studies. Ann N Y Acad Sci. 1990;600:649-61; discussion 661-4. doi: 10.1111/j.1749-6632.1990.tb16916.x. PMID: 1979216.

[3]Mamounas LA, Mullen CA, O'Hearn E, Molliver ME. Dual serotoninergic projections to forebrain in the rat: morphologically distinct 5-HT axon terminals exhibit differential vulnerability to neurotoxic amphetamine derivatives. J Comp Neurol. 1991 Dec 15;314(3):558-86. doi: 10.1002/cne.903140312. PMID: 1814975.

[4]Brown P, Molliver ME. Dual serotonin (5-HT) projections to the nucleus accumbens core and shell: relation of the 5-HT transporter to amphetamine-induced neurotoxicity. $\quad J \quad$ Neurosci. 2000;20(5):1952-1963. doi:10.1523/JNEUROSCI.20-05-01952.2000.

[5]Furlong TM, Leavitt LS, Keefe KA, Son JH. Methamphetamine-, d-Amphetamine-, and p-Chloroamphetamine-Induced Neurotoxicity Differentially Effect Impulsive Responding on the Stop-Signal Task in Rats. Neurotox Res. 2016;29(4):569-582. doi:10.1007/s12640-016-9605-9

[6]Da Prada M, Picotti GB. Content and subcellular localization of catecholamines and 5-hydroxytryptamine in human and animal blood platelets: monoamine distribution between platelets and plasma. Br J Pharmacol. 1979;65(4):653-662. doi:10.1111/j.1476-5381.1979.tb07878.x

[7]Bacqué-Cazenave J, Bharatiya R, Barrière G, et al. Serotonin in Animal Cognition and Behavior. Int J Mol Sci. 2020;21(5):1649. Published 2020 Feb 28. doi:10.3390/ijms21051649

[8]Dalley J.W., Everitt B.J., Robbins T.W. Impulsivity, compulsivity, and top-down cognitive control. Neuron. 2011;69:680-694. doi: 10.1016/j.neuron.2011.01.020.

[9]Grant J.E., Kim S.W. Brain circuitry of compulsivity and impulsivity. CNS Spectr. 2014;19:21-27.

[10]Ouzir M, Errami M. Etiological theories of addiction: A comprehensive update on neurobiological, genetic and behavioural

vulnerability. Pharmacology Biochemistry and Behavior. 2016;148:59-68. doi: 10.1016/j.pbb.2016.06.005.

[11]Verdejo-García A, Lawrence AJ, Clark L. Impulsive behavior as a vulnerability marker for substance-use disorders: review of findings from high-risk research, problem gamblers and genetic association studies. Neuroscience \& Biobehavioral Reviews. 2008;32:777-810. doi: 10.1016/j.neubiorev.2007.11.003.

[12]Whiteside SP, Lynam DR. The Five Factor Model and impulsive behavior: Using a structural model of personality to understand impulsive behavior. Personality and Individual Differences. 2001;30:669-689. doi: 10.1016/S0191-8869(00)00064-7.

[13]Ersche KD, et al., Drug addiction endophenotypes: impulsive versus sensation-seeking personality traits. Biol Psychiatry, 2010. 68(8): p. 770-3.

[14]Verdejo-Garcia A, Lawrence AJ, and Clark L, Impulsivity as a vulnerability marker for substance-use disorders: review of findings from high-risk research, problem gamblers and genetic association studies. Neurosci Biobehav Rev, 
2008. 32(4): p. 777-810.

[15]Kozak K, Lucatch AM, Lowe DJE, Balodis IM, MacKillop J, George TP. The neurobiology of impulsivity and substance use disorders: implications for treatment. Ann N Y Acad Sci. 2019;1451(1):71-91. doi:10.1111/nyas.13977

[16]Cammisuli DM, Innocenti A, Franzoni F, Pruneti C. Aerobic exercise effects upon cognition in Mild Cognitive Impairment: A systematic review of randomized controlled trials. Arch. Ital. Biol. 2017;155:54-62.

[17]Abrantes AM, Blevins CE. Exercise in the context of substance use treatment: key issues and future directions. Curr Opin Psychol. 2019 Dec;30:103-108.

[18]Dolezal BA, Chudzynski J, Storer TW, et al. Eight weeks of exercise training improves fitness measures in methamphetamine-dependent individuals in residential treatment. J Addict Med. 2013;7:122-128.

[19]Rawson RA, Chudzynski J, Gonzales R, et al. The impact of exercise on depression and anxiety symptoms among abstinent methamphetamine-dependent individuals in a residential treatment setting. J Subst Abuse Treat. 2015a;57:36-40.

[20]Rawson RA, Chudzynski J, Mooney L, et al. Impact of an exercise intervention on methamphetamine use outcomes post-residential treatment care. Drug Alcohol Depend. 2015b;156:21-28.

[21]Wang D, Zhu T, Zhou C, Chang YK. Aerobic exercise training ameliorates craving and inhibitory control in methamphetamine dependencies: a randomized controlled trial and event-related potential study. Psychol Sport Exerc. 2017;30:82-90.

[22]Fisher BE, Petzinger GM, Nixon K, et al., 2004. Exercise-induced behavioral recovery and neuroplasticity in the 1-methyl-4-phenyl-1,2,3,6-tetrahydropyridine-lesioned mouse basal ganglia. J Neurosci Res, 77: 378-90

[23]Winstanley CA, Olausson P, Taylor JR, Jentsch JD. Insight into the relationship between impulsivity and substance abuse from studies using animal models. Alcoholism: Clinical and Experimental Research. 2010;34:1306-1318.

[24]Kirby LG, Zeeb FD, Winstanley CA. Contributions of serotonin in addiction vulnerability. Neuropharmacology. 2011;61(3):421-432. doi:10.1016/j.neuropharm.2011.03.022

[25]Stanford M, Mathias C, Dougherty D, Lake S, Anderson N, Patton J. Fifty years of the Barratt impulsiveness scale: an update and review. Personal Individ Differ. 2009;47(5):385-395. doi: 10.1016/j.paid.2009.04.008.

[26]Hassani-Abharian P,Ganjgahi H, Tabatabaei-Jafari H, et al., 2015. Exploring Neural Correlates of Different Dimensions in Drug Craving Self-Reports among Heroin Dependents[J]. Basic Clin Neurosci 6(4): 271-284.

[27]Ren J, Friedmann D, Xiong J, Liu CD, Ferguson BR, Weerakkody T, et al. . Anatomically defined and functionally distinct dorsal raphe serotonin sub-systems. Cell. (2018) 175:472-87.e20.

[28]Ohmura Y, Tsutsui-Kimura I, Sasamori H, Nebuka M, Nishitani N, Tanaka KF, et al. Different roles of distinct serotonergic pathways in anxiety-like behavior, antidepressant-like, and anti-impulsive effects. Neuropharmacology. (2019) 9:107703 10.1016/j.neuropharm.2019.107703

[29]Nordin C, Sjodin I. CSF monoamine patterns in pathological gamblers and healthy controls. Journal of Psychiatic Research. 2006;40:454-459. 
[30]Marazziti D, Golia F, Picchetti M, Pioli E, Mannari P, Lenzi F, Conversano C, Carmassi C, Catena Dell'Osso M, Consoli G, Baroni S, Giannaccini G, Zanda G, Dell'Osso L. Decreased density of the platelet serotonin transporter in pathological gamblers. Neuropsychobiology. 2008;57:38-43.

[31]Da Prada M, Picotti GB, 1979. Content and subcellular localization of catecholamines and 5-hydroxytryptamine in human and animal blood platelets: monoamine distribution between platelets and plasma. Br J Pharmacol 65, 653-662. [32]Audhya T, Adams JB, Johansen L, 2012. Correlation of serotonin levels in CSF, platelets, plasma, and urine. Biochim Biophys Acta 1820, 1496-1501.

[33]Sarrias MJ, Cabre P, Martinez E, Artigas F, 1990. Relationship between serotoninergic measures in blood and cerebrospinal fluid simultaneously obtained in humans. J Neurochem 54, 783-786.

[34]Huang J, Zheng Y, Gao D, Hu M, Yuan T. Effects of Exercise on Depression, Anxiety, Cognitive Control, Craving, Physical Fitness and Quality of Life in Methamphetamine-Dependent Patients. Front Psychiatry. 2020;10:999. Published 2020 Jan 28. doi:10.3389/fpsyt.2019.00999

[35]Rawson RA, Chudzynski J, Gonzales R, et al. The Impact of Exercise On Depression and Anxiety Symptoms Among Abstinent Methamphetamine-Dependent Individuals in A Residential Treatment Setting. J Subst Abuse Treat. 2015;57:36-40. doi:10.1016/j.jsat.2015.04.007

[36]Dolezal BA, Chudzynski J, Dickerson D, et al. Exercise training improves heart rate variability after methamphetamine dependency. Med Sci Sports Exerc. 2014;46(6):1057-1066. doi:10.1249/MSS.0000000000000201

[37]Alberghina D, Giannetto C, Piccione G. Peripheral serotoninergic response to physical exercise in athletic horses. $J$ Vet Sci. 2010;11(4):285-289. doi: $10.4142 /$ jvs.2010.11.4.285 